19 Revue d'histoire du XIXe siècle

Société d'histoire de la révolution de 1848 et des

révolutions du XIXe siècle

$24 \mid 2002$

Varia

\title{
La frontière (le voisin) et l'étranger. Les enjeux identitaires d'un conflit frontalier
}

\section{Laurent Dornel}

\section{OpenEdition \\ Journals}

Édition électronique

URL : http://journals.openedition.org/rh19/372

DOI : $10.4000 /$ rh19.372

ISSN : $1777-5329$

Éditeur

La Société de 1848

Édition imprimée

Date de publication : 1 juin 2002

Pagination : 11-124

ISSN : 1265-1354

Référence électronique

Laurent Dornel, « La frontière (le voisin) et l'étranger. Les enjeux identitaires d'un conflit frontalier». Revue d'histoire du XIXe siècle [En ligne], 24 | 2002, mis en ligne le 07 mars 2008, consulté le 20 avril 2019. URL : http://journals.openedition.org/rh19/372 ; DOI : 10.4000/rh19.372

Ce document a été généré automatiquement le 20 avril 2019

Tous droits réservés 


\title{
La frontière (le voisin) et l'étranger. Les enjeux identitaires d'un conflit frontalier
}

\author{
Laurent Dornel
}

1 «Tracer une frontière, écrit Étienne Balibar, c'est précisément définir un territoire, le délimiter, et ainsi enregistrer son identité ou la lui conférer ${ }^{1}$. La frontière, clôture symbolique et limite physique du territoire, définit une appartenance et une exclusion: c'est un des éléments fondamentaux de la distinction entre le Nous et le Non-Nous ${ }^{2}$, ou, pour reprendre la terminologie de Norbert Elias, entre les Outsiders et les Established. Là, peut-être plus qu'ailleurs, à l'échelle locale donc, se définit l'altérité, joue de manière complexe le lien entre identité et territoire. En somme, la frontière est un de ces lieux où se forge l'identité nationale, où, par conséquent, se matérialise l'opposition entre le national et l'étranger. Longtemps, celui qui vit de l'autre côté de la frontière est considéré comme voisin et adversaire à la fois ; mais au XIX ${ }^{\mathrm{e}}$ siècle, il tend à devenir avant tout un étranger, rejeté et stigmatisé, et pourtant indispensable à la définition, par les populations françaises, de leur propre identité. L'essor, dans la seconde moitié du siècle, de l'immigration, ainsi que l'amputation de 1870, contribuent à durcir la représentation de l'étranger, désormais de plus en plus clairement identifié. Le propos de cet article est d'observer, à partir de l'analyse des conflits frontaliers dans la vallée pyrénéenne de Baïgorry, le rapport dialectique voisin/étranger, rapport qui se radicalise à mesure que se durcissent les frontières de la France ${ }^{3}$.

De toutes nos frontières, celle des Pyrénées a sans doute été la moins contestée : cette chaîne montagneuse (Roger Dion parle de la "barrière des Pyrénées " ${ }^{4}$ ), limite naturelle entre deux territoires, a été assez tôt transformée en frontière militaire, "ethnique " (Dion) puis politique, pour devenir une « frontière immobile " 5 . Mais nous savons que les vallées - d'un même versant comme de versants opposés - depuis le XIII ${ }^{\mathrm{e}}$ siècle au moins, ont passé entre elles des conventions afin d'assurer " paix et bon voisinage ${ }^{6}$ et de régler les problèmes de compascuité (ou vaine pâture) : ce sont les fameux traités de lies et 
passeries ${ }^{7}$, ou encore faceries. Ces conventions, décidées localement sans intervention étatique ${ }^{8}$, ont été confirmées, plus ou moins intégralement, par les traités francoespagnols, du Traité des Pyrénées en 1659 à ceux de Bayonne en 1856-1858. Elles ont permis l'établissement et le maintien de relations globalement pacifiques, fondées sur le partage des ressources écologiques (eau, forêts, estives...). Au-delà de cette stabilité d'ensemble, la frontière des Pyrénées, au XIX ${ }^{e}$ siècle, fut presque continûment agitée par des rivalités entre communautés françaises et espagnoles, par des différends locaux interminables pouvant dégénérer en micro-crises violentes et nécessiter l'intervention arbitrale des deux États ${ }^{9}$. Afin de mieux cerner le rôle de la frontière dans la production de l'étranger, nous avons choisi d'étudier l'un de ces conflits durables dont la rémanence permet de nous inscrire dans la longue durée : il s'agit du conflit qui opposa, dans le sud de la vallée de Baïgorry, les communautés française et espagnole, à propos d'une zone appelée Pays Quint. Ce petit territoire appartenait à l'État espagnol, mais était depuis des décennies concédé aux Baïgorriens qui l'exploitaient moyennant une redevance annuelle ${ }^{10}$.

3 Ce différend ne date pas du XIX siècle; mais il acquiert alors une importance et une intensité nouvelles. En mai 1829, le procureur général de Pau rend compte au Garde des Sceaux de l'enlèvement, sur le territoire espagnol, de quatre-vingts vaches appartenant à des bergers français ${ }^{11}$. Les menaces de l'administration française pour que les bêtes soient restituées n'y ont rien fait. Du coup, les bergers français ont monté une expédition pour récupérer ces dernières, ce qui a envenimé les relations entre les deux parties. Mais, écrit le procureur, «nos français n'ont été amenés à se faire justice eux-mêmes que par suite des vexations journalières des Espagnols qui abusent de l'existence d'un traité dont nous réclamons depuis longtemps la modification ${ }^{12}$. On soulignera au passage le possessif «nos» du procureur; il est l'expression d'un paternalisme qui n'est pas sans évoquer l'Ancien Régime ("nos sujets»), mais surtout, il identifie très nettement les Baïgorriens : aux yeux de la puissance publique, les populations des frontières sont incontestablement françaises.

4 En 1840, entreprenant une démarche collective, les maires et adjoints des communes de La Fonderie, Ascarat, Lasse, Anhaux, Irouléguy, Saint-Étienne, des Aldudes, ainsi que des "habitants sachant signer», adressent aux députés une pétition de douze pages ${ }^{13}$ : le différend frontalier y est représenté comme « une question vitale pour la vallée et dans laquelle sont gravement engagés les intérêts matériels et la dignité de la France ». Les pétitionnaires attirent l'attention des députés sur «le désordre moral qu'entretiennent aux Aldudes principalement, cette incertitude sur la nationnalité [sic] et sur le respect dû aux Lois dont la violation est journalière et flagrante, la nécessité d'être toujours prêt à recourir aux armes pour repousser les violences et les outrages, et enfin la répression inefficace des crimes et des délits par suite du conflit des deux juridictions ».

5 L'objet précis du litige est un "vaste terrain (environ dix lieues carrées) en nature de pâturage et de bois connu sous le nom d'Aldudes ou Pays quint ${ }^{14}$ [dont] les deux vallées ont joui par indivis de temps immémorial ", cette jouissance commune étant garantie par des « contrats de faceries et compascuités perpétuelles » ${ }^{15}$. Il s'agit donc en quelque sorte de ce que Roger Dion a appelé un "désert-frontière " ${ }^{16}$. Pendant des décennies, l'équilibre démographique entre les deux communautés française et espagnole a assuré à la vallée un certain calme. Mais l'essor de la population du côté français rompt cet équilibre, en rendant nécessaires des défrichements ${ }^{17}$ vers la partie méridionale de la vallée (en direction d'Erro). Cette rupture d'équilibre a deux conséquences. 
6 Tout d'abord, de nouvelles communautés apparaissent : les pétitionnaires rappellent en effet, que «les français mêlés à quelques espagnols formèrent donc les villages de La Fonderie et des Aldudes peuplés aujourd'hui d'environ 4500 habitants, et les Espagnols celui de Val Carlos dont la population est d'environ 900 habitants». Ainsi, selon les plaignants, les villages qui se disputent férocement quelques lieues carrées ont une origine commune. De fait, comme ailleurs ${ }^{18}$ dans les Pyrénées, les différences entre communautés situées de part et d'autre de la frontière, sont minimes : les conditions de vie, les activités économiques, la culture, la langue même (le catalan, le basque), sont quasiment similaires. Comme l'a montré Sahlins pour la Cerdagne ${ }^{19}$, la différenciation vient avant tout de l'appartenance à deux États différents, qui se traduit par des stratégies personnelles ou collectives antagonistes. Le fait que les habitants, par leur pétition, en appellent aux députés, c'est-à-dire à la représentation nationale, est l'une des expressions de ces stratégies.

7 D'autre part, dès le XVII ${ }^{e}$ siècle, les conflits provoqués par l'extension des défrichements ont suscité l'intervention des deux gouvernements, dont la mission était de faire respecter l'ordre: des conventions ont été signées, notamment en 1614 et 1715, le traité de 1785 a mis un terme aux conflits par le partage du terrain litigieux, en fixant « la ligne séparative tout à la fois du territoire des deux royaumes et des droits sur le quint des vallées copropriétaires ». Cette décision royale a été immédiatement contestée par les Français, qui perdaient alors «toutes les positions militaires, le village français d'Endarrole et les mines de fer et de cuivre qu'il contient ». Mais surtout, elle « enlevait aux communautés françaises, sans leur aveu, sans les avoir entendues, les pâturages les plus précieux, indispensables, pour élever leurs bestiaux qui forment leur unique moyen d'existence ». Néanmoins, la Révolution et l'Empire ont «oublié » de faire exécuter le traité, si bien qu'à la Restauration, "les deux vallées sont rentrées dans la jouissance indivise qui s'est continuée jusqu'en 1827 ». Ces interventions du pouvoir central, quoique peu suivies d'effets rigoureux, ont rapproché cependant les communautés villageoises de l'État et de ses représentants civils (préfet et sous-préfet) ou judiciaires (procureur du roi, procureur général). L'adresse aux députés est à cet égard significative de la permanence et du resserrement de ce lien entre la " périphérie » et le « centre ».

8 C'est à la fin des années 1820 que les hostilités reprennent : le concessionnaire des mines de Baïgorry fait imprudemment commencer une coupe de bois sur le versant méridional, ce qui entraîne la riposte immédiate des Espagnols, qui demandent l'application du traité de 1785, requête à laquelle le ministre français accède. Cette décision française évidemment suscite l'ire des habitants du versant français, qui doivent consentir «à payer une redevance conditionnelle pour continuer à jouir des pâturages indispensables à la conservation de leurs troupeaux ${ }^{20}$. Les gouvernements acceptent de reprendre les discussions, mais celles-ci traînent en longueur. En 1830, les choses se précipitent: les pasteurs français cessent de payer la redevance tout en faisant paitre leurs animaux sur les herbages litigieux. Et lorsque les Espagnols saisissent une partie des troupeaux qu'ils parquent dans le village de Burguette, les Baïgorriens lèvent 1200 hommes pour récupérer les bêtes. Là-dessus, éclate la révolution de Juillet, qui dote la France d'un "gouvernement national », lequel obtient de l'Espagne « le rétablissement provisoire de la jouissance indivise des pâturages par les deux vallées ».

9 Mais les habitants de Baïgorry ne sont pas au bout de leur peine, puisqu'une guerre civile éclate en Navarre, qui déstabilise la région : maraudeurs et soldats font leur apparition, si bien que « les bestiaux [sont] enlevés, les bergers maltraités, leurs cabanes pillées ». En 
1838, alors qu'avancent les troupes carlistes, les femmes et les enfants habitant la partie espagnole se réfugient "en France ", tandis que les hommes "se renfermèrent et furent assiégés dans un fort qui ne pouvait être avantageusement attaqué sans violation du territoire français. Pendant le siège, ils reçurent de France tous leurs approvisionnements de guerre et de bouche ».

Cette solidarité de circonstance ne met pas fin aux anciennes rivalités, qui reprennent. Le nouvel ambassadeur à Madrid, Rumigny, alerte le ministre des Affaires étrangères, en vain. La colère à nouveau s'empare des habitants français de la vallée, mais cette fois elle se manifeste contre le gouvernement : « La générosité est un noble sentiment, sans doute, et qui distingue la nation française entre toutes les autres, mais aussi elle lui a souvent coûté cher. Et ici, propice à l'Espagnol, elle deviendrait cruauté, déni de justice pour nous français spoliés de nos droits, toujours inquiets sur la conservation de nos moyens d'existence, à la merci nos biens [sic] et nos personnes de voisins haineux et barbares ». L'analyse de ces épisodes permet de mettre en valeur plusieurs phénomènes.

11 Face à un ennemi nouveau, extérieur à la vallée, il semble donc que les deux communautés passent une sorte d'alliance, renouent avec des solidarités anciennes. Ce comportement social fait songer aux alliances et rivalités (feud) qu'Evans-Pritchard a étudiées chez certains nilotes ${ }^{21}$ : le schéma par lequel il formalise les échelles identitaires (p. 170) permet un parallèle anthropologique. Les tribus sont composées de segments qui s'opposent les uns aux autres, mais aussi, dans certains cas, s'unissent face à d'autres segments ou tribus : « un segment tribal est un groupe politique par rapport à d'autres segments du même genre, et ils forment ensemble une tribu, mais par leur seul rapport à d'autres tribus nuer, et à des tribus étrangères adjacentes, qui forment autant de parties du même système politique $~^{22}$. Un système (c'est-à-dire selon Evans-Pritchard, un "ensemble de relations entre groupes») politique peut donc être défini, ainsi que le souligne Louis Dumont dans la préface de l'ouvrage, comme un «système de groupes territoriaux » (p.IV). Ce que montre ici l'anthropologue, c'est que le lien avec l'Autre, qu'il soit amical ou agonistique, est nécessaire à l'existence d'un groupe, qu'il est l'une des conditions de la définition de soi. Ces interrelations, sur lesquelles Norbert Elias a tant insisté, sont marquées par une agressivité quasi permanente, par des motifs de querelle : « une dispute à propos d'une vache ; [...] le droit à un point d'eau lors de la saison sèche ; les droits de pâture [...] ${ }^{23}$.

12 Les habitants des Vallées n'ont cessé de se quereller, parfois très violemment, en particulier dans le cadre de la lutte pour le contrôle des ressources écologiques (eau, pâtures, estives...), mais n'en n'ont pas moins, depuis le Moyen Âge, conclu des accords, les fameuses lies et passeries. En fait, ces interrelations entre communautés pyrénéennes ont conduit à la construction d'un étranger nécessaire, mais un étranger familier, bien circonscrit, auquel s'oppose un étranger plus lointain, abstrait, menaçant. On songe ici à la distinction établie dans certaines régions françaises entre les petits étrangers et les grands étrangers, les premiers étant définis avant tout par le critère parental (système lignager), les seconds par le critère spatial ${ }^{24}$. Fréquemment en conflit avec son alter ego français, le paysan pyrénéen espagnol partage pourtant avec lui un même territoire, souvent un même mode de vie. Cette identité, liée à une inscription dans un espace sinon similaire du moins ressemblant, l'emporte encore, dans le premier tiers du XIX ${ }^{\mathrm{e}}$ siècle, sur les différences construites (i.e. nationalité). C'est pourquoi en 1838, les habitants français de la vallée vont même jusqu'à approvisionner ceux de la partie espagnole, engagés dans une lutte avec des Espagnols mais qui n'appartiennent pas à la vallée. 
13 Mais, et c'est tout de même pour nous l'essentiel, cette opposition entre communautés est renforcée et matérialisée par la frontière, sur laquelle se cristallisent des enjeux qui transcendent l'espace local.

Pour les habitants des vallées de Baïgorry et d'Erro, la frontière, en temps de guerre notamment, produit des effets juridiques et politiques avec lesquels ils jouent : pour se protéger, les Espagnols se réfugient en effet dans un fort que seule une violation du territoire français permettrait de prendre. La frontière, par conséquent, n'est pas qu'une ligne imaginaire, imposée par un pouvoir central qui serait seul producteur d'identité. Elle sépare en même temps qu'elle met en relation; elle entre constamment dans les relations entre des communautés tantôt rivales et ennemies, tantôt alliées. Elle définit une zone d'échanges certes instable, mais caractérisée par des intérêts communs.

Devant la menace d'un tiers, les communautés habituellement rivales s'entraident : ici, l'identité l'emporte. Mais en temps normal, c'est la concurrence et l'altérité qui dominent. Or, objectivement, les deux communautés en présence sont très proches, socialement, culturellement et économiquement. Elles doivent donc produire l'altérité et la différenciation nécessaires à la conscience de soi comme groupe. Dans le dernier extrait cité, on observe ce double mouvement d'affirmation identitaire et de différenciation. Dans un premier temps, les pétitionnaires se revendiquent comme français (« la nation française », « nous français »), mettent en doute ce qu'on appellerait aujourd'hui le «nationalisme» du gouvernement, et en appellent «à la représentation nationale mieux éclairée et encore plus jalouse du bien-être et de l'honneur de tous les Français ». Dans un deuxième temps, ils stigmatisent le voisin qui est désormais "l'Espagnol », " haineux et barbare». On notera ici l'emploi de l'adjectif «barbare», symbole même de cette différenciation. On est en présence du diptyque «nous » et « les autres» (ou: "les hommes» et les "barbares»), analysé par les anthropologues, de Marcel Mauss ${ }^{25}$ à Ralph David Grillo. Ce dernier, dans l'introduction à l'ouvrage qu'il dirige, écrit : "a contrast within nature itself may be utilized, as when We are of one blood, but They are beasts ${ }^{26}$. La frontière permet de définir les insiders et les outsiders, ceux qui « en sont » et ceux qui « n'en sont pas », c'est-à-dire les étrangers.

Dans le cas que nous analysons, les pétitionnaires parlent donc de "barbares", terme qui exprime toute la distance qui les sépare de leurs voisins. Plus à l'est, vers Oloron, il arrive que l'on se traite mutuellement de Gabatcho ou Gavatxos. Cette injure s'entend plus loin encore: les Catalans l'emploient pour désigner les non Catalans et en particulier les Occitans ${ }^{27}$. Cette épithète révèle à elle seule l'ambiguïté des relations frontalières : c'est un dérivé de gaba (goitre ${ }^{28}$, gésier, gorge) qui habituellement s'emploie à l'égard de l'étranger familier. En ancien provençal, gavach désigne l'étranger, le rustre, le montagnard; les pasteurs espagnols traitent donc leurs homologues français de «montagnards », ce qui en soi est juste! Mais le terme, par extension, désigne aussi toute personne d'origine étrangère et, en Espagne, il est employé pour qualifier, de façon dépréciative, les Français. Les injures entre villages proches et rivaux sont chose fréquente dans la France tout entière. Dans le Pays-Haut lorrain, lougnad est le terme dialectal (qui signifie: une personne qui regarde en dessous, sournoise) par lequel on désigne celui d'en face ${ }^{29}$. Mais la présence de la frontière modifie la portée de l'injure. Selon Peter Sahlins, qui le rencontre en Cerdagne, gavatcho, terme péjoratif, est « l'indice d'une plus grande adéquation entre la frontière politique et la frontière ethnique entre les deux peuples $»^{30}$. 
17 Dans l'immédiat, cette pétition de 1840 n'a pas modifié la situation. Mais le Traité des Limites du 2 décembre 1856 octroie aux habitants de Baïgorry « la jouissance exclusive et perpétuelle des pâturages » pour la partie septentrionale des Aldudes et, pour le versant méridional (au cœur des conflits) une jouissance des pâturages « en union avec les Espagnols " ${ }^{31}$. Sur ce dernier, les bergers français sont donc considérés comme des " étrangers de passage » en Espagne, bien qu'il leur soit accordé des droits d'exemption et de construction de cabanes. Toutefois, au cours des années suivantes, bien que ses données globales soient modifiées, le conflit ne cesse pas.

18 Le 4 mai 1873, le syndic de Saint-Étienne-de-Baïgorry se plaint au Préfet de ce que le Pays Quint soit « devenu entièrement espagnol par le dernier traité de délimitation entre la France et l'Espagne, si défavorable non seulement aux intérêts baïgorriens mais encore aux intérêts français " ${ }^{32}$. Le 2 mai 1882 , le syndic ${ }^{33}$ de la vallée de Baïgorry cette fois adresse au Préfet une lettre dans laquelle il prévient ce dernier que face à l'inaction du gouvernement, les habitants sont prêts à "recourir à des résolutions extrêmes "; précisément, est envisagé l'envoi de «vingt jeunes gens armés» pour soutenir les pasteurs français et «les défendre contre les entreprises des agents du gouvernement espagnol et ceux des vallées adverses ${ }^{34}$. Cette lettre témoigne de l'achèvement du processus de différenciation entre les villages et hameaux des vallées: l'Autre est clairement identifié comme l'adversaire, pasteurs et carabiniers sont rejetés sous la même opprobre. Telle est en tout cas la perception que les Baïgorriens veulent officiellement laisser paraître. Les échanges ancestraux ont-ils pour autant disparu? Probablement pas, mais le contexte politique et culturel dans lequel ils se déroulent désormais s'est modifié. Le règlement du conflit intervient dans les mois suivants : le 7 août 1882, le ministre des Affaires étrangères écrit au ministre de l'Intérieur : «Je suis heureux de vous faire savoir que cette affaire parait avoir reçu une solution favorable. Le gouvernement espagnol, en effet, avait promis à notre ambassadeur d'envoyer les instructions nécessaires pour que les habitants de Baïgorry pussent conduire librement leurs troupeaux dans les pâturages concédés: comme d'autre part, les plaintes de nos nationaux ne se sont pas renouvelées, [...] il y a lieu de croire que le Cabinet de Madrid a tenu ses engagements ». De fait, le 29 août, est promulgué un règlement, signé par le marquis de la Vega de Armijo, ministre d'État. Il y est stipulé que les habitants des deux pays peuvent et doivent laisser paître les troupeaux dans le Pays Quint, « sans s'assujettir à aucune formalité administrative ou fiscale ». Ce qui change néanmoins, c'est que les entrées et sorties de bestiaux doivent avoir lieu en présence des autorités espagnoles, des carabiniers notamment.

19 Jusqu'au milieu du siècle au moins, en Baïgorry notamment, les échanges entre les vallées françaises et espagnoles étaient réglés par des accords ancestraux, d'où les États étaient absents. Dans cette société de face à face, l'Autre était à la fois le voisin et l'étranger, l'adversaire et l'allié, selon les circonstances. L'étranger apparaît par conséquent comme une nécessité anthropologique, à laquelle se surimpose cependant, au fil des ans, une autre construction politique, mais aussi juridique : la nationalité. L'affirmation de la frontière, locale et nationale, qui exprimait l'identité des communautés, est peu à peu officialisée en quelque sorte, notamment à partir des années 1880, par la propagande nationaliste, qu'elle soit le fait des gouvernements ou celui de "partis», ligues, associations, etc. Peu à peu, sont politisés et nationalisés les conflits qui auparavant étaient plus étroitement circonscrits à l'échelle locale. Ce qui pour les villageois du début du siècle n'était qu'une intrusion dans un territoire devient alors une violation du 
territoire national. La présence accentuée de l'État en ses périphéries, la nationalisation de la frontière, le renforcement du lien juridique entre l'État et les individus consécutivement à la redéfinition de la nationalité ${ }^{35}$, l'intégration ${ }^{36}$ accélérée des campagnes les plus enclavées dans la nation française expliquent probablement, chez ces populations frontalières pastorales et plus généralement rurales, une conscience de soi plus accrue, plus nette, comme membre d'une "communauté imaginée " ${ }^{37}$. La frontière tend à faire disparaître l'entre-soi qui, malgré tout, a longtemps caractérisé les populations limitrophes. Du coup, l'étranger est beaucoup mieux distingué et apparaitt comme radicalement différent de soi.

Les conflits et les stratégies autour de la frontière finissent par accompagner ou produire ce qu'on appelle un «sentiment national», parfois plus intense que celui que véhicule l'État central. La frontière et le territoire alentour sont considérés comme dignes de l'intérêt national: «nos besoins et notre patriotisme, écrivent les pétitionnaires de la vallée de Baögorry en 1840, nous faisaient un devoir de montrer à vos regards, Messieurs, une limite territoriale qui désertant les positions avantageuses où la plaçaient la nature et la raison, dans l'intérêt de la défense du pays, descend en vue et tout près du clocher de Notre-Dame des Aldudes et permet à l'Espagne, en cas d'aggression [sic], de saccager le village avant qu'on n'y soit averti du danger ». Cette notion d'intérêt, que l'on retrouve à plusieurs reprises dans le cas que nous étudions, paraît centrale. En effet, si le nationalisme, comme le rappelle Ernest Gellner ${ }^{38}$ est "essentiellement un principe politique", il s'exprime aussi par un ensemble d'interactions et de comportements sociaux, ou encore par la défense d'intérêts spécifiques. Dans le cas présent, on observe une liaison de plus en plus étroite entre les "intérêts baïgorriens" et les "intérêts français ", c'est-à-dire entre les intérêts particuliers d'une communauté et ceux de l'État, auquel est reconnu désormais le monopole de la violence. Le sentiment nationaliste n'est donc pas seulement un sentiment "pur ": il prend également en charge les aspects pratiques de la construction ou de la défense d'un territoire national.

21 Pour attirer l'attention du pouvoir central et obtenir gain de cause, les villageois, qui se doutent bien que de simples querelles pastorales ne sauraient intéresser les députés, tentent d'extraire leurs revendications du cadre étroitement local dans lequel elles s'insèrent en jouant sur l'aspect stratégique de la frontière: ainsi les espaces qu'ils défendent si âprement ne sont-ils plus seulement territoire villageois, mais désormais territoire national. La défense de ce dernier les place du même coup dans une situation de défenseurs de la nation. Comme dans le cas de la Cerdagne, les rivalités entre communautés ont permis, en définissant radicalement les "gens du dehors», la cristallisation et l'imprégnation d'un sentiment d'appartenance nationale. Si les communautés villageoises de la vallée de Baïgorry, à l'instar de celles de Cerdagne, ont, par leurs querelles incessantes avec les communautés espagnoles, apporté « la nation au village ${ }^{39}$, elles ont dans le même temps hissé le village au niveau de la nation. Comme dans la pétition de 1840 , les Baïgorriens, dont les problèmes fondamentalement n'ont pas changé (ils vivent surtout de l'élevage, ce qui rend nécessaires les terrains de pâture), tentent de lier leurs intérêts à ceux de la nation. Mais alors qu'au début du siècle les deux communautés demeuraient malgré tout assez proches et liées, dans les années 1870, les rivalités se sont figées, la frontière s'est pétrifiée, tandis que le mouvement d'intégration nationale s'est accéléré sous l'effet des progrès ferroviaires, économiques (constitution d'un marché national) et culturels (développement de l'instruction avec Duruy). De plus, la guerre de 1870 et la perte par la France de l'Alsace et de la Lorraine ont développé la 
«sensibilité nationale». Le syndic de la vallée de Baïgorry attire ainsi l'attention du Préfet sur un point qui n'est pas vraiment lié au contrôle des ressources écologiques : «les Espagnols ont élevé sur cette partie deux grandes casernes, où sont installés d'ordinaire les carabiniers. En cas de guerre, Urepel, les Aldudes, presque toute la vallée, seraient à la merci de l'ennemi, qui aurait les facilités les plus grandes pour un coup de main ». La menace, qui restait dans la pétition de 1840 assez vague, se fait ici beaucoup plus précise, d'autant qu'elle est matérialisée par la présence de " casernes ». S'il est clair que les Baïgorriens se livrent à une forme de manipulation en exagérant le danger, on ne peut exclure totalement que cette manipulation recouvre un « sentiment national », réel, voire exacerbé. L'exemple de la Cerdagne, non loin de là, à peu près à la même époque, permet à Peter Sahlins de conclure que «les démonstrations les plus virulentes du nationalisme se situent à l'extrême périphérie, où l'opposition entre la France et l'Espagne est la plus saillante, et où le militarisme du discours local atteint parfois l'injure $»^{40}$. Ce militarisme s'exprime en Baïgorry dans la même lettre du 4 mai 1873 : en effet, outre la révision du traité de 1856, le syndic réclame l'établissement d'un poste de soldats français pour les pasteurs et que le nombre de gardes-limites soit doublé.

Le contexte historique dans lequel s'inscrit cette affaire a été progressivement modifié par l'engagement croissant des représentants locaux du pouvoir central en faveur des communautés villageoises. Ainsi le sous-préfet de Mauléon regrette-t-il, le 12 avril 1874, l'opposition de la Junte royale de Navarre (tenue par les carlistes en quête de reconnaissance officielle) à des pourparlers entre les vallées d'Erro et Baztan d'une part, et le syndic de Baïgorry d'autre part ${ }^{41}$. Le Préfet écrit au ministre de l'Intérieur, deux jours plus tard: "cette question des pâturages est vitale, pour nos vallées basques si tristement sacrifiées dans les derniers traités avec l'Espagne; le mécontentement des habitants sera immense et l'émigration, qui dépeuple véritablement le département, prendra de nouvelles proportions, si elle ne reçoit pas une solution favorable ${ }^{42}$. Plus loin, il propose, en quelque sorte à titre compensatoire, d'assurer les élus de «la sollicitude très vive du gouvernement pour les intérêts de nos nationaux et [de] ses efforts persévérants pour les défendre ».

En 1881, les habitants de Baïgorry introduisent une réclamation relative à l'exercice du droit de pâturage dans le Pays Quint. Le 21 avril, le préfet rapporte au ministre de l'Intérieur que « depuis le mois de novembre dernier, l'Administration espagnole a mis certaines entraves à l'exercice des droits que les traités internationaux ${ }^{43}$ ont conférés [sic] aux habitants de la vallée française de Baïgorry pour la jouissance des pâturages de leurs troupeaux dans le Pays Quint ${ }^{44}$. Ici, même si l'émotion est vive dans la vallée de Baïgorry, ce ne sont plus deux communautés qui s'opposent, mais deux États. Le préfet, à la fin de son rapport, demande au Ministre de lui «faire connaître quelle devrait être notre attitude vis-à-vis des autorités espagnoles dans le cas où elles persévéreraient dans leurs exigences et dans la menace de la suppression ou de l'amoindrissement de nos droits ». L'emploi du pronom personnel «nos » non seulement confirme l'implication du Préfet, incarnation de l'autorité du gouvernement et défenseur de la souveraineté nationale, mais encore souligne la dimension nationale des enjeux locaux. Les Baïgorriens n'ont désormais plus besoin d'attirer l'attention du gouvernement: la sollicitude de ce dernier se manifeste naturellement. L'acuité de la perception de la frontière s'exprime à tous les niveaux. Mais elle n'empêche pas les surenchères et les manipulations: la périphérie continue de témoigner au centre les marques d'un dépit intéressé. 

de pair avec une modification de la perception de l'Autre, avec qui les liens traditionnels ont tendance à s'estomper: celui d'en face, qui était ensemble l'adversaire et l'allié, devient progressivement l'étranger ennemi. De plus en plus, les populations frontalières manifestent clairement leur identité nationale, sans pour autant abandonner une forte identité locale: ainsi, les disputes, querelles et rixes ne doivent pas être entendues comme de simples anecdotes; elles « amènent [les communautés villageoises] à adopter de nouvelles identités nationales sans renoncer à leurs identités locales et le fait d'exclure les étrangers vivant en leur sein revient [...] à affirmer leur propre nationalité ${ }^{45}$. Incidemment, ce qui se passe à la frontière montre la complexité de l'élaboration et de la diffusion du «sentiment national»: nos Baïgorriens, comme les Cerdans de Sahlins, se livrent constamment à des manipulations identitaires, un jour requérant l'intervention et l'aide du gouvernement, l'autre s'opposant à lui et lui témoignant sa défiance. L'identité nationale, à la frontière, apparaît dès lors comme un processus qui résulte de relations dialectiques entre le centre et la périphérie. Plus les années passent, plus les formes idéologiques imposées par le centre se développent, bouleversant ainsi le fragile équilibre des interrelations frontalières: la frontière devient un enjeu national. S'y exprime désormais à chaque moment la souveraineté du territoire national, souveraineté dont les gouvernements, la presse, le personnel politique et les populations frontières, surtout après 1870, se montrent les défenseurs sourcilleux: la surveillance des frontières est renforcée, bénéficiant pour cela des progrès des techniques d'identification. Dans la xénophobie des années 1880 et 1890, marquée notamment par le thème de l'invasion, la frontière tient une place non négligeable, car elle est la digue dont la rupture menace la France tout entière ${ }^{46}$.

La frontière, à mesure qu'elle se matérialise et se politise, apparaît de plus en plus irrémédiablement liée à la nation; elle n'est plus seulement limite, mais poste avancé, ligne - en voie de fortification - protégeant le territoire national, définissant ainsi un " chez nous » et un au-delà menaçant, pays des étrangers. D'une certaine manière, elle radicalise les identités et définit, dans une nécessaire et complémentaire relation, le national et l'étranger, l'inclus et l'exclus. Les conflits frontaliers apparaissent en dernier ressort comme des constructions politiques, car ils mettent en scène non plus seulement des rivalités que l'anthropologue reconnait comme constitutives des sociétés rurales, mais des oppositions que structure (ou qui structurent) efficacement ce que l'on désigne habituellement par « sentiment national ». Les frontières constituent un enjeu national, politique et idéologique essentiel parce qu'elles touchent à la souveraineté territoriale, à la mystique nationale ${ }^{47}$; elles participent activement à l'évolution de la catégorie d'étranger et, ainsi, contribuent à la construction d'une identité nationale longtemps imprécise. Parfois même, comme dans le cas de la vallée de Baïgorry où la nationalité est restée longtemps un critère de distinction tout à fait secondaire, on peut voir les populations frontalières produire de l'extranéité, s'affirmer dans une volonté de différenciation. L'éloignement par rapport au centre, loin de se traduire par quelque irrédentisme, suscite au contraire un fort attachement à la patrie. En somme, si l'étranger n'existait pas, il faudrait l'inventer... Par ailleurs, l'analyse de ces conflits semble mettre en cause le «modèle statocentré de construction identitaire ${ }^{48}$ : longtemps on a cru en 
France que l'identité nationale découlait d'abord de l'État central, irradiant ensuite progressivement les marges, ainsi révélées à la Nation. Depuis quelques années déjà, un certain nombre de travaux nuancent fortement l'idée d'une modernisation politique liée à une "descente de la politique vers les masses " ${ }^{49}$ et celle d'une nationalisation très tardive de la société française ${ }^{50}$. Christine Guionnet, analysant les élections municipales sous la monarchie de Juillet, montre que, malgré les limites rencontrées par l'autorité étatique, « ce régime constitue une période d'accentuation de la construction symbolique du national au niveau communal ${ }^{51}$ et souligne que les élections ont permis une véritable problématisation de l'identité communautaire [...] premier pas vers l'avènement d'un individu se pensant comme sujet politique ${ }^{52}$. Autrement dit, ainsi que le souligne Pierre Rosanvallon dans la préface à cet ouvrage, «le développement de l'État-nation et de l'appartenance à celui-ci se réalise, tout au moins sous la période étudiée, dans l'imbrication entre la nation et le local et non par l'éradication du second au profit du premier $"{ }^{53}$. Dans les Pyrénées, les rapports entre la nation et le village, ou plus globalement entre le centre et la périphérie, ont été diversement analysés. Pour JeanFrançois Soulet, le XIX ${ }^{e}$ siècle fait reculer considérablement la société pyrénéenne traditionnelle; face aux "agressions multiformes" dont elles sont l'objet, les communautés pyrénéennes déploient un certain nombre « d'actions contestataires » qui traduisent "un désaccord général avec les forces étatiques (socio-économiques, culturelles) dominantes» au point que l'on peut parler d'une "population en dissidence ${ }^{54}$. Autrement dit, la périphérie résiste au centre. Mais des travaux récents apportent un autre point de vue. Peter Mc Phee ${ }^{55}$ estime que le rattachement précoce du Roussillon au territoire national s'explique en grande partie par «la conquête républicaine dont les petites villes et les campagnes constituent les théâtres privilégiés »; il observe, à l'occasion de la Révolution de 1848, une mobilisation politique sans précédent, qui initie l'enracinement d'une tradition républicaine. Certes, la frontière est marquée par l'autorité étatique (augmentation des contrôles douaniers et renforcement des polices frontalières, affectation de fonctionnaires énergiques, affirmation du rôle politique et militaire de Perpignan); néanmoins, cette francisation d'une terre catalane est indissociable d'un fort engagement politique, d'un apprentissage collectif du républicanisme. Peter Sahlins, pour la Cerdagne, va plus loin : pour lui, la nation émerge à la périphérie avant même qu'elle ne soit imposée par le centre et avant l'application des politiques d'intégration nationale par les deux États concernés.

26 L'image d'un État s'affirmant contre les périphéries, détruisant les autonomies et caractères locaux, revêtant par conséquent un caractère en quelque sorte intrinsèquement répressif doit être mise en cause au profit d'une conception plus dialectique et dynamique ${ }^{56}$ : les rapports entre les marges et le pouvoir central sont beaucoup plus nuancés, complexes et complémentaires, en particulier au $\mathrm{XIX}^{\mathrm{e}}$ siècle, époque décisive pour le processus de nationalisation de la société française. La construction politique des identités, qui accompagne le lent passage de la communauté à la société ${ }^{57}$, ne peut se concevoir dans la seule opposition perpétuelle des populations au pouvoir étatique. 


\section{NOTES}

1.. Étienne BALIBAR, La Crainte des masses. Politique et philosophie avant et après Marx, Éditions Galilée, 1997, 456 p., p. 372.

2.. Nous empruntons cette expression à Clifford GEERTZ, Works and lives. The anthropologist as author, Cambridge, Polity Press, 1988, traduction française Ici et là-bas. L'anthropologue comme auteur, Éditions Métailié, 1996, 152 p.

3.. Cet article s'appuie sur des recherches menées dans le cadre plus général d'une thèse : Laurent DORNEL, La France hostile. Histoire de la xénophobie en France au XIX ${ }^{e}$ siècle, thèse d'histoire sous la direction de Gérard Noiriel, École des hautes études en sciences sociales, 2001, 3 volumes, $731 \mathrm{f}^{\circ}$.

4.. Roger DION, Les frontières de la France, Paris, Éditions Hachette, 1979 (1 1 ère édition 1947), $110 \mathrm{p}$.

5.. L'expression est de Daniel NORDMAN et Jacques REVEL, dans André BURGUIERE [dir.], Histoire de la France, Éditions du Seuil, 1989, p. 42. Daniel Nordman a repris et développé ses travaux sur la frontière dans un ouvrage récent : Daniel NORDMAN, Frontières de France. De l'espace au territoire, XVI ${ }^{e}$-XIXe siècle, Éditions Gallimard, 1998, 644 p. Le XIX siècle n'y est cependant abordé que de façon marginale. Pour une histoire de la frontière pyrénéenne, on consultera Jean SERMET, La frontière hispano-française des Pyrénées et les conditions de sa délimitation, Tarbes, Les Amis du Livre Pyrénéen, 1983, 285 p. ; et Carlos de Fernandez de Casadevante ROMANI, La frontière franco-espagnole et les relations de voisinage, avec une référence spéciale au secteur frontalier du Pays Basque, Bayonne, Éditions Harriet, $1989,453 \mathrm{p}$.

6.. Jean SERMET, La frontière hispano-française..., ouv. cité., p. 195.

7.. Xavier de PLANHOL, Géographie historique de la France, Librairie Arthème Fayard, 1988, 635 p., p. 140.

8.. D'après le juriste Descheemaeker, ces accords entre vallées françaises mêmes et avec les vallées espagnoles, signifient que « la notion de frontière " leur était étrangère ; Prénom ? DESCHEEMAEKER, La frontière pyrénéenne de l'Océan à l'Aragon, Paris, 1945 [cité par Jean SERMET, La frontière hispano-française..., ouv. cité, p. 195].

9.. Sur ce point, voir Laurent DORNEL, La France hostile..., ouv. cité, f ${ }^{\circ}$ 512-522. Pendant tout le XIX ${ }^{e}$ siècle, les frontières sont le lieu de rivalités, de tensions et de rixes entre les populations. Ces phénomènes, dont les manifestations vont croissant, recoupent plusieurs types de conflits. Il s'agit pour l'essentiel de rivalités pastorales, de contrebande et de braconnage, de provocations diverses témoignant d'un sens de plus en plus aigu de la nationalité de chacun. Ces rivalités ne sont pas propres aux zones de confins : Robert MUCHEMBLED pour l'époque moderne et Frédéric CHAUVAUD pour le XIX ${ }^{e}$ siècle ont montré qu'elles font partie de ces rapports sociaux marqués par une violence omniprésente ; Robert MUCHEMBLED, La violence au village. Sociabilité et comportements populaires en Artois du XVe au XVII e siècle, Paris, Éditions Brepols, 1989, 419 p. ; Frédéric CHAUVAUD, Les passions villageoises au XIX ${ }^{e}$ siècle. Les émotions rurales dans les pays de Beauce, du Hurepoix et du Mantois, Paris, Éditions Publisud, 1995, 272 p. Mais, dans le cas de la vallée de Baïgorry, la présence de la frontière les radicalise. 
10.. Pour une histoire du Pays Quint, on se reportera à Jean SERMET, « La question du Pays Quint ", dans Jean SERMET, La frontière hispano-française..., ouv. cité, chapitre 6. 11.. Arch. nat. (archives nationales), BB18 1173.

12. Le traité en question est celui d'Elizondo (1785), dont le but était de « consolider encore et encore les liens d'amitié et de parenté qui unissent si étroitement les deux souverains et de voir leurs vassaux jouir de cette bonne harmonie... » ainsi que de « déplacer et ôter tous les motifs de dissensions et de plaintes qui subsistent entre les frontaliers des monts Pyrénées ». Il concernait plus spécifiquement les vallées frontières espagnoles du Baztan, Erro, Valcarlos et Roncevaux, et françaises de Baïgorry, St-JeanPied-de-Port et Cize (voir Carlos de Fernandez de Casadevante ROMANI, La frontière francoespagnole..., ouv. cité, pp. 93-94).

13.. Arch. nat., C 2179. Ce type d'archive est particulièrement intéressant dans la mesure où le regard officiel - présent dans la plupart des séries d'archives (administratives, policières, voire judiciaires) - laisse ici la place à une prise de parole par les individus eux-mêmes. Cette parole, si elle demeure contrôlée ne serait-ce que par le statut des scripteurs, n'en échappe pas moins aux filtres habituels du discours étatique.

14.. Cette zone se situe plus précisément de la partie méridionale de la vallée de Baïgorry, seule partie qui fut l'objet de litiges. Cette partie de la vallée (les Aldudes) fut défrichée au cours des siècles par les Baïgorriens, qui n'hésitèrent donc pas à envahir les territoires incontestablement espagnols de Roncevaux et d'Erro (Jean SERMET, La frontière hispanofrançaise..., ouv. cité, p. 199). Voir aussi Daniel NORDMAN, Frontières de France, ouv. cité, pp. 332 et suivantes.

15.. Arch. nat. F7 12578, Extrait des registres des délibérations du Conseil général des Basses-Pyrénées, Session du 29 novembre 1871.

16.. Roger DION, Les frontières de la France, ouv. cité., p. 11.

17.. Jean SERMET, La frontière hispano-française..., ouv. cité, p. 208.

18. Pour le Roussillon, voir par exemple Geneviève GAVIGNAUD, « La frontière pyrénéenne et la partie française de la Catalogne depuis 1659 », dans Frontières et limites de 1610 à nos jours, Actes du $101^{e}$ congrès national des sociétés savantes, Lille, 1976, Section d'histoire moderne et contemporaine, tome 1, Bibliothèque nationale, 1978, 328 p., pp. 155-170.

19.. Peter SAHLINS, Boundaries : the making of France and Spain in the Pyrenees, Berkeley, University of California Press, 1989, traduction française Frontières et identités nationales. La France et l'Espagne dans les Pyrénées depuis le XVII siècle, Paris, Éditions Belin, 1996, 416 p.

20.. Arch. nat., C 2179, ainsi que les citations qui suivent.

21.. Edward E. EVANS-PRITCHARD, The Nuer, a description of the modes of livelihood and political institutions of a Nilotic people, Oxford, Clarendon Press, 1940, traduction française Les Nuer. Description des modes de vie et des institutions politiques d'un peuple nilote, Paris, Éditions Gallimard, 1968, 312 p. On aurait pu évoquer les pages que Marcel Mauss consacre au potlatch, « système de prestations sociales » qui se caractérise lui aussi par des « formes agonistiques où des individus et des groupes viennent absorber ou détruire les richesses du clan à la fois opposé et allié » [c'est nous qui soulignons] ; Marcel MAUSS, « Don, contrat, échange », dans Marcel MAUSS, Euvres, Paris, Éditions de Minuit, 1969, tome 3, pp. 39-32. Le recours à la sociologie et à l'anthropologie n'est pas décoratif : inscrit dans la démarche générale de nos recherches, caractéristique de la socio-histoire à laquelle nous nous rattachons, il fournit à l'historien des outils pour analyser le rapport à l'Autre, qui est au cœur de notre problématique. Enfin, ce recours se justifie ici par l'exemplarité de la situation de la vallée de Baïgorry d'une part, et d'autre part, par 
l'importance sur d'autres frontières des rituels que nous étudions (sur ce dernier point, voir notre thèse).

22.. Edward E. EVANS-PRITCHARD, The Nuer..., ouv. cité, p. 175.

23.. Norbert ELIAS, Die Gesellschaft der Individuen, Francfort-sur-le-Main, Suhrkamp, 1987, traduction française, La Société des individus, Paris, Librairie Arthème Fayard, 1991, 301 p., p. 178. Voir aussi, dans ce même ouvrage, ce qu'il nomme « chaînes invisibles ", « réseau de dépendances », ou encore " interdépendance fonctionnelle ».

24.. Voir en particulier Claude KARNOOUH, «L'étranger ou le faux inconnu. Essai sur la définition spatiale d'autrui dans un village lorrain », dans Ethnologie française, tome 2, n - 1-2, 1972, pp. 107-121.

25.. Marcel MAUSS, « Objet, méthodes et divisions de l'ethnologie », dans CEuvres, ouv. cité, p. 314.

26. Ralph David GRILLO, « Nation » and «State » in Europe. Anthropological Perspectives, Londres, Academic Press, 1980, 201 p., p. 13.

27.. Voir Geneviève GAVIGNAUD, « La frontière pyrénéenne... », art. cité.

28.. Le goitre étant une maladie fréquente chez les montagnards ; voir Alain REY [dir.], Dictionnaire historique de la langue française, Paris, Éditions du Dictionnaire Le Robert, 1992, 2 volumes.

29.. Claude KARNOOUH, « L'étranger ou le faux inconnu... », art. cité, p. 115 ; Voir aussi Frédéric CHAUVAUD, Les passions villageoise..., ouv. cité, p. 19-24.

30.. Peter SAHLINS, Frontières et identités nationales..., ouv. cité., p. 249.

31.. Carlos de Fernandez de Casadevante ROMANI, La frontière franco-espagnole..., ouv. cité, p. 179.

32.. Arch. nat., F7 12578.

33.. Syndicat pastoral formé en 1837 et reconnu par une Ordonnance royale en 1838 ; voir Jean SERMET, La frontière hispano-française..., ouv. cité, p. 194.

34.. Arch. nat., F7 12577.

35.. La nationalité désigne, en droit, l'appartenance d'une personne à un État, et non, comme on le croit souvent, à une nation. Sur ce point, Gérard NOIRIEL, État, nation et immigration. Vers une histoire du pouvoir, Éditions Belin, 2001, 400 p., plus précisément le chapitre $5:$ « Socio-histoire d'un concept : les usages du mot "nationalité" au XIX ${ }^{e}$ siècle ».

36.. Nous employons ce terme dans le sens que lui a conféré Norbert ELIAS, La Société des individus, ouv. cité, en particulier la $3^{\mathrm{e}}$ partie : « Les transformations de l'équilibre "nousje" ».

37.. Benedict ANDERSON, Imagined Communities, Londres, Verso, 1983, traduction française L'imaginaire national. Réflexions sur l'origine et l'essor du nationalisme, Paris, Éditions de La Découverte, 1991, 213 p.

38.. Ernest GELLNER, Nations et nationalisme, Éditions Payot, 1989 (1983), 208 p., p. 11. La phrase complète est : « le nationalisme est essentiellement un principe politique qui affirme que l'unité politique et l'unité nationale doivent être congruentes ». 39.. Bernard LEPETIT, « préface » dans Peter SAHLINS, Frontières et identités nationales..., ouv. cité, p. 7.

40.. Peter SAHLINS, Frontières et identités nationales..., ouv. cité, p. 248.

41.. Arch. nat., F7 12578, Rapport au Préfet des Basses-Pyrénées.

42.. Arch. nat., F7 12578.

43.. Il s'agit du Traité des Limites de décembre 1856, par lequel la propriété du Pays Quint revient aux vallées espagnoles de Baztan et d'Erro. 
44.. Arch. nat., F7 12577.

45.. Peter SAHLINS, Frontières et identités nationales..., ouv. cité., p. 302.

46. L'intensification de la xénophobie que nous avons observée au cours de ces années coïncide avec une vague protectionniste sans précédent. Parfois, la frontière est littéralement fermée. En 1890, c'est le cas pour la frontière pyrénéenne, officiellement pour des motifs sanitaires ; en réalité, comme en 1821, il s'agit surtout de limiter la mendicité étrangère. Fermer la frontière donne donc à l'État français les moyens de mieux contrôler l'afflux des étrangers, d'en repousser certaines catégories (les libéraux en 1821, les mendiants en 1890) et d'instaurer des procédures d'identification. Renforcer les contrôles à la frontière, c'est faire le tri entre les étrangers, partant, protéger la nation contre ceux que l'on appellera sous peu les « indésirables ».

47. Voir par exemple, après 1870 « la ligne bleue des Vosges » que Maginot transforme en ligne fortifiée en 1930.

48. L'expression est de Bernard LEPETIT, « Préface », art. cité.

49. Voir Maurice AGULHON, La République au village. Les populations du Var de la Révolution à la Seconde République, Paris, Éditions du Seuil, 1979, 543 p.

50.. Voir Eugen WEBER, Peasants into Frenchmen, Stanford University Press, Stanford, 1976, traduction française La Fin des terroirs. La modernisation de la France rurale 1870-1914, Librairie Arthème Fayard, 1983, 839 p.

51.. Christine GUIONNET, L'apprentissage de la politique moderne. Les élections municipales sous la monarchie de Juillet, Paris, Éditions L'Harmattan, 1997, 324 p., pp. 263-264.

52.. Idem, p. 298.

53.. Idem, p. iv.

54.. Jean-François SOULET, « Une nouvelle approche de la France rurale au XIX ${ }^{\mathrm{e}}$ siècle ? À propos de thèses récentes sur les Pyrénées ", dans Revue Historique, 1989, 2, pp. 381-392 ; voir aussi Jean-François SOULET, Les Pyrénées au XIX ${ }^{e}$ siècle, Toulouse, Éditions Eché, 1987, 2 volumes, $478 \mathrm{p}$. et $713 \mathrm{p}$.

55.. Peter MC PHEE, Les Semailles de la République dans les Pyrénées-Orientales, 1846-1852, Perpignan, Publications de L'Olivier, 1995, 509 p.

56.. Voir notamment de Jean-François CHANET, L'École républicaine et les petites patries, Paris, Éditions Aubier, 1996, 427 p. L'auteur montre que le processus qui a mené à une relative uniformisation linguistique du pays est bien plus complexe et nuancé et ne peut se réduire à l'image d'une République imposant par la force et les brimades la langue française au détriment des dialectes.

57.. Sur ce point, voir Ferdinand TÖNNIES, Gemeinschaft und Gesellschaft: Grundbegriffe der reinen Soziologie, Berlin, Éditions Curtius, 1912, traduction française Communauté et société. Catégories fondamentales de la sociologie pure, Éditions Retz-CEPL, 1977. Pour Christine GUIONNET, le conflit politique, lié à la pluralité des clans impulsée par les élections, peut être considéré comme facteur clé d'une construction identitaire, mais aussi comme moment où la société s'institue. En effet, ce conflit est à l'origine de « nouveaux groupes identitaires politiquement constitués et dictincts des identités sociales liées au vécu quotidien, au sein d'une société n'acceptant qu'une représentation des identités données » ; voir Christine GUIONNET, L'apprentissage de la politique moderne..., ouv. cité, p. 303. 


\section{RÉSUMÉS}

Les populations frontalières, dans les Pyrénées notamment, ont entretenu entre elles d'une part, avec le pouvoir étatique d'autre part, des relations complexes, à la fois de proximité et de confiance, de distance et de méfiance. Tel est le cas des communautés paysannes françaises et espagnoles de la vallée de Baïgorry, longtemps peu différenciées, mais dont les liens au XIXe siècle se transforment à la faveur de la nationalisation de la société française. Les conflits pastoraux, anciens et ritualisés, mettent en scène des communautés aux identités de plus en plus différenciées ; en raison d'une modification globale de la perception de la frontière, leurs enjeux dépassent désormais le cadre local. Ces conflits sont ici l'occasion de s'interroger sur cette présence renouvelée de la nation aux marges : s'agit-il d'une présence imposée par le "centre" en dépit des résistances locales, ou bien d'un processus plus complexe, n'excluant pas, de la part des populations concernées, un certain nombre de manipulations identitaires?

The border (the neighbour) and the foreigner. The identity stakes of a border conflict. The border populations, especially in the Pyrenees, maintained complex relationships with one another as well as with state power. It was a mixture of proximity and trust, of distance and suspicion. That was particularly the case of the Spanish and French rural communities in the valley of Baïgorry, which had been very similar for centuries, but whose relations changed in the $19^{\text {th }}$ century with the nationalisation of French society. The old and ritualised, pastoral conflicts now opposed communities whose identities were increasingly different. Because of a global change in the perception of the border, their goals now transcended the local setting. These conflicts enable us to question this renewed presence of the nation on its fringes: was it something imposed by the central state despite local resistance or was it a more complex process which did not exclude a certain amount of identity manipulations from the involved populations?

\section{AUTEUR}

\section{LAURENT DORNEL}

L'auteur est professeur en lycée, chercheur associé au Centre interdisciplinaire de recherches et d'études sur les juifs et les disporas (CIREJD), CNRS-Université Toulouse 2-Le Mirail. 